Eur. J. Clin. Chem. Clin. Biochem.

Vol. 30, 1992, pp. $753-759$

C) 1992 Walter de Gruyter \& Co. Berlin · New York

\title{
Mucin-Like High Molecular Mass Protein Fractions from Total Pig Gallbladder Bile Mucus, Pig Gallbladder Wall Mucus, and Total Human Gallbladder Bile Mucus
}

\author{
By Frauke Sabinski ${ }^{1}$, U. Wosiewitz ${ }^{1}$ and U. Leuschner ${ }^{2}$ \\ ${ }^{1}$ Institute of Medical Physics and Biophysics, Westfälische Wilhelms-Universität Münster \\ ${ }^{2}$ Center of Internal Medicine, Dept. Gastroenterology, Johann Wolfgang Goethe Universität Frankfurt/Main, \\ Germany
}

(Received February 6/June 19, 1992)

\begin{abstract}
Summary: Native mucin-like complexes were obtained from both pig gallbladder bile and pig gallbladder wall mucus by precipitation, centrifugation, and gel permeation chromatography. Crude preparations by either dialysis (native mucus from bile, and native gallbladder wall mucus) or by precipitation (crude total bile mucus, and lipomucoid) were purified by gel permeation chromatography on Sephacryl S-300HR and Sephacryl S-500HR (Pharmacia). The elution profiles obtained with a reversibly denaturing and detergentcontaining eluent showed the same pattern for all samples, although the amounts of the four main fractions differed somewhat. The excluded fraction with the highest carbohydrate portion had an apparent $M_{\mathrm{r}}>10^{7}$. This fraction and the following included lipomucoid (in physiological solution tightly bound to fraction I), and an eluent-insoluble mucus portion from all samples were characterized by determination of the protein concentration, carbohydrates, sialic acids, and lipids, using standard methods. Sugar analysis was performed by gas-liquid chromatography.
\end{abstract}

Human gallbladder bile was subjected to the same procedures of mucus precipitation and separation. Human gallbladder bile mucus showed identical behaviour to that of pig gallbladder bile mucus, and showed a very similar elution pattern in gel chromatography.

\section{Introduction}

In 1967 Nakamura (1) demonstrated, by histological staining, the presence of an organic network of acid mucopolysaccharides throughout $\mathrm{CaCO}_{3}$ stones. Since then, highly glycosylated material or periodateSchiff (PAS)-reacting complexes have been recovered in mammalian (including human) biles, and isolated from human gallstones $(2-6)$. Furthermore, animal experiments and investigations on stone patients revealed an enhanced mucus production, often preceding the formation of gallstones $(7-10)$.

This has been taken as an indication that biliary mucins promote the pathological process $(11-14)$. In addition, since mucus becomes a part of the stone structure, it is likely to influence chemolitholysis, as it is not dissolvable in organic litholysis media like monooctanoin or methyl-t-butyl ether (27). Moreover, in chemolitholysis, stone debris, consisting of calcium bilirubinate, bilirubin polymers, inorganic calcium salts and mucoprotein, is left behind within the gallbladder. At this stage, and with respect to an adjuvant mucolytic therapy, a thorough knowledge of the mucus chemistry is needed, as well as information on the relationship of mucus with other bile constituents. For the experimental elucidation of the role of biliary mucus in both lithogenesis and chemolitholysis, adequate model material is needed, as well as the appropriate methodology for its isolation and characterization. Attempts to separate mucus from bile only deal with readily soluble mucus fractions, i.e. fractions soluble in distilled water or saline, and 
they exclude insoluble portions and naturally occurring complexes which do not consist of mucus entirely. In fact, the solubility of mucus has often been found to be rather poor (15), because it tends to form heterogeneous macromolecular complexes $(10,16)$ and/or firmly binds to other proteins, bile pigments and lipids by $\mathrm{H}$-bridges and by Van der Waals forces $(3,5,17,18)$. Pig gallbladder bile mucin, which has been reported to be quite similar to human bile mucin (2), and which is amply and easily available, was used to develop an adequate methodology for the isolation of mucoprotein complexes in a preparative scale.

The developed methods were applied to human bile, and an approach was made to further characterize the isolated porcine material.

\section{Materials and Methods}

\section{Material}

Pig gallbladders were obtained from the local slaughterhouse immediately after slaughtering. Human bile was aspirated intraoperatively by gallbladder punction and stored at $-20^{\circ} \mathrm{C}$. Pig gastric mucin, sialic acid, and bovine serum albumin were purchased from Sigma. Dodecyl N,N-dimethylammonio-3-propane sulphonate (SB12) was a product of Serva. All reagents were analytical grade.

\section{Preparation procedures}

The preparation scheme is shown in figure 1.

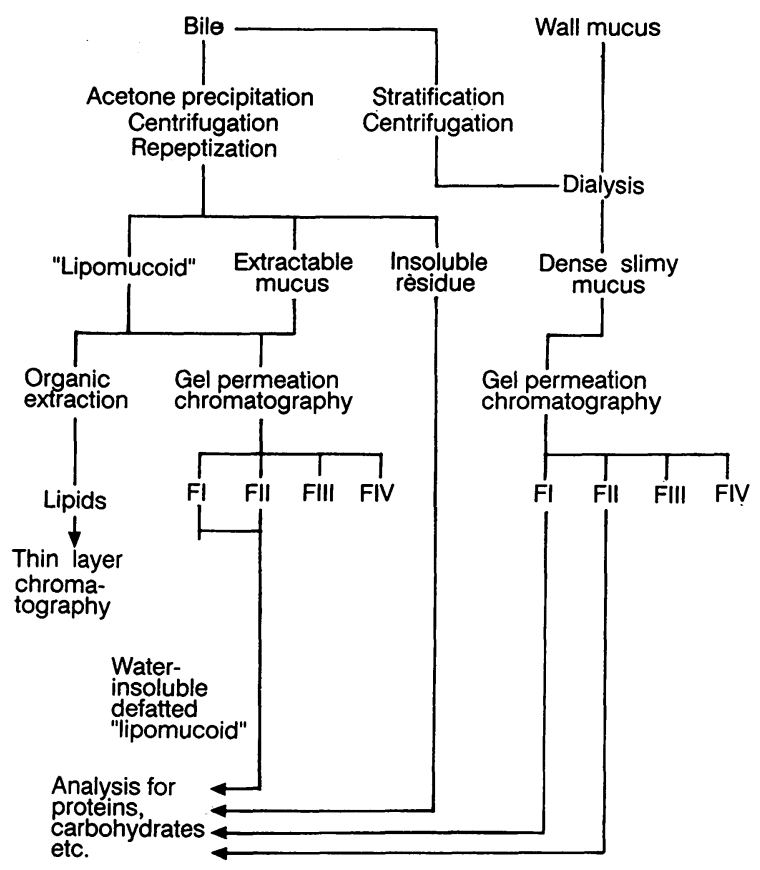

Fig. 1. Processing of pig gallbladder bile mucus and pig gallbladder wall mucus.

\section{Native mucus}

Pig gallbladders were cleaned from adherent liver tissue, rinsed with tap and deionized water, and stored on crushed ice. Each bladder was suspended at the $D$. cysticus stump, and the bile was carefully aspirated with a syringe. Individual biles were pooled. After the addition of ascorbic acid $(2 \mathrm{~g} / \mathrm{l}$, sodium azide $0.4 \mathrm{~g} / \mathrm{l})$, and phenylmethyl sulphonylfluoride $(1 \mathrm{mmol} / \mathrm{l})$ the samples were cooled in an ice-water bath and stored at $4{ }^{\circ} \mathrm{C}$ for $48 \mathrm{~h}$. The emptied bladders were sectioned longitudinally, and clamped on a support while the remaining bile was removed thoroughly by rinsing with deionized water. The viscous wall mucus was gently scraped off with a soft-edged plastic spatula. The mucus was pooled, homogenized, centrifuged for $30 \mathrm{~min}$ at $1100 \mathrm{~g}$, and dialysed against deionized water $(+0.4 \mathrm{~g} / \mathrm{l} \mathrm{Na}$ azide) at $4{ }^{\circ} \mathrm{C}$. The pooled bile fluid batches which had undergone stratification during storage at $4{ }^{\circ} \mathrm{C}$ for $48 \mathrm{~h}$, were separated into a liquid upper bile layer and a dense slimy mucus zone. The latter was homogenized, centrifuged for $30 \mathrm{~min}$ at $1100 \mathrm{~g}$, thoroughly dialyzed against distilled water $(0.4 \mathrm{~g} / 1 \mathrm{Na}$ azide), rehomogenized, and stored at $4{ }^{\circ} \mathrm{C}$.

\section{Acetone-precipitated material}

Bile batches that did not show a distinct tendency to stratify (plus the liquid upper layer, mentioned above) were overlayered with ice-cold acetone (volume fraction up to 0.50 ) and gently stirred to quantitatively precipitate crude bile mucus. The yellowish pellet, separated by centrifugation $(20 \mathrm{~min}, 1100 \mathrm{~g})$, was washed with cold aqueous acetone (volume fraction 0.50 ) until the supernatant became clear and colourless. Acetone was substituted stepwise by buffered physiological saline $(\mathrm{NaCl} 7 \mathrm{~g} / \mathrm{l}$, ascorbic acid $2 \mathrm{~g} / \mathrm{l}, \mathrm{Na}$-azide $0.4 \mathrm{~g} / \mathrm{l}$, dissolved in $2 \mathrm{mmol} / \mathrm{l}$ $\mathrm{KNaHPO}_{4}, \mathrm{pH}$ 7.9). The light-grey pellets from the centrifugation steps were covered by floating lipid-rich upper layers, which were collected separately and designated 'lipomucoid'. The pellets of crude mucus were suspended in buffered physiological saline (see above) and vigorously shaken for $20 \mathrm{~h}$ at room temperature in the dark. After centrifugation $(30 \mathrm{~min}$, $1100 \mathrm{~g}$ ) the supernatant was collected and the shaking procedure was repeated until redissolvable material could no longer be extracted. The insoluble residue was thoroughly washed with deionized water and freeze-dried.

Lipomucoid was liberated from non-covalently bound lipids by exhaustive extraction with

(a) chloroform : methanol $2: 1$, and $1: 2$, respectively;

(b) chloroform : methanol : water $1: 2: 0.8$;

(c) chloroform : methanol : buffered physiological saline 1.7 : $3.3: 5$ (all mixtures $\mathrm{v} / \mathrm{v})$.

The organic extracts were pooled, concentrated by vacuum evaporation, dried over sodium sulphate and subjected to thin layer chromatography as described elsewhere (19). The waterinsoluble defatted lipomucoid was desalted by washings with distilled water and finally freeze-dried.

Human and porcine gallbladder bile, both having been stored at $-20^{\circ} \mathrm{C}$ until use, were thawed, homogenized, centrifuged, and subjected to acetone precipitation as described above. The redissolved mucus from human and from pig gallbladder bile was subjected to gel permeation chromatography.

\section{Gel permeation chromatography}

This method was performed on Sephacryl S-300HR and S500HR (Pharmacia) using an eluent containing urea $(2 \mathrm{~mol} / \mathrm{l})$, SB12 $(10 \mathrm{~g} / 1)$, and Na-azide $(0.4 \mathrm{~g} / \mathrm{l})$. The $\mathrm{pH}$ was kept at 7.0. Samples were equilibrated in the eluent, saturated with $\mathrm{Na}$ tetraborate and centrifuged for $5 \mathrm{~min}$ at $10500 \mathrm{~g}$ prior to chro- 
matography. The fractions were pooled, concentrated by freezedrying, redissolved and dialysed against distilled water. The dialysate again was freeze-dried, and residual SB12 was removed from the solids by ethanol extraction.

Analyses of protein content, carbohydrates and lipids

Protein was measured by the method of Onishi et al. (20). Glycoprotein fractions were determined according to De Jong et al. (21). Sialic acids were quantified with the ninhydrin test as described by Yao \& Ubuka (22) and with the periodateresorcinol method, respectively, as described by Jourdian et al. (23). Monosaccharides and fatty acids were measured by capillary gas-liquid chromatography (24). Carbohydrates were calculated from the results of the monosaccharide assay. Pig gastric mucin (PSM, Sigma) was treated likewise to affirm the results.

Semiquantitative, 1-dimensional thin layer chromatography (19) on precoated silica gel plates (Merck) was performed to obtain information on those lipids associated with the mucus.

\section{Results}

\section{Preparation procedures}

In order to recover a sufficient quantity of mucus, 7 preparation cycles were performed, each on the pooled gallbladder biles of $15-30$ animals $(700-$ $1500 \mathrm{ml}$ ). The seven batches differed from each other in their consistency (viscosity, readiness to stratify). Freeze-stored porcine and human bile, when thawed, did not differ from fresh bile in consistency and colour.

\section{Dialysis method}

After homogenization of the dialysate the yellowbrown native crude mucus formed a turbid, slightly opalescent fluid, stable for $\sim 2$ month when stored at $4{ }^{\circ} \mathrm{C}$. Bile samples, in which mucus had not been preconcentrated gelatinously by spontaneous stratification, could not be dialysed; they formed a precipitate of pigment-protein polymers.

\section{Precipitation method}

When acetone was used to separate crude mucus from bile, most of the bile salts, lecithins and bile pigments remained dissolved in the discarded supernatant. There was no evidence of any denaturation or breakdown of the mucus by the organic solvent: during gel permeation chromatography the crude total bile mucus preparation behaved like the native mucus preparation from bile. Unlike the pale precipitates produced when fresh bile was treated with acetone, the precipitates from human and porcine biles, which had been stored in a deeply frozen state, were deeply coloured by pigments even after numerous washings with aqueous acetone, volume fraction 0.5 .

\section{Gel permeation chromatography}

Sephacryl S-300HR chromatography of the fractions always produced the same peak pattern, comprising four main fractions (peak/fraction I-IV, see fig. 2). The ratios of the individual peak integrals, however, showed moderate variations.

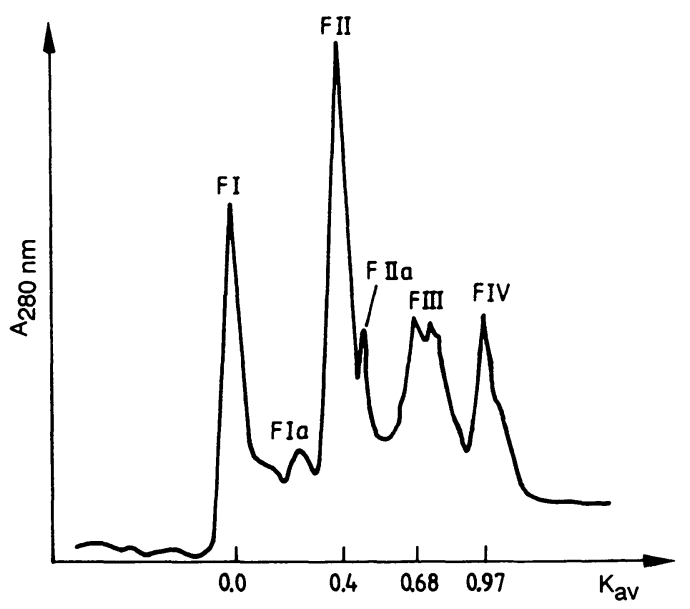

Fig. 2. Gel permeation elution profile. Stationary phase: Sephacryl S-300 HR

Eluent: $2 \mathrm{~mol} / \mathrm{l}$ urea, $10 \mathrm{~g} / \mathrm{l} \mathrm{B12}$.

Sample: Extractable mucus.

Column: $77 \mathrm{~cm}, \mathrm{ID}=1.6 \mathrm{~cm}$.

Flow rate: $16 \mathrm{ml} / \mathrm{h}$.

Fraction I, which was eluted with the void volume both on Sephacryl S-300HR and S-500HR, was the most sensitive fraction when subjected to the glycoprotein test. It was considered to be the intrinsic bile mucus fraction (see also 1.c. $(2,3,5,25,26))$ and its apparent $M_{\mathrm{r}}$ was $>10^{7}$, according to the exclusion limits of the stationary phases $\left(>10^{6}\right.$ and $\left.2 \times 10^{7}\right)$. Dextran blue, taken as a calibration standard $\left(M_{\mathrm{r}} \mathrm{Q} 2\right.$ $\times 10^{6}$ ), was included by S-500HR while fraction I was eluted with the void volume as a sharp, symmetrical peak (not presented).

A weak positive carbohydrate reaction was observed with fraction II $\left(\mathrm{K}_{\mathrm{av}}=0.4\right)$. This fraction, accompanied by some pigment (and therefore pale green of colour), was co-eluted with fraction I when distilled water, physiological or $2 \mathrm{~mol} / \mathrm{l}$ saline or buffered physiological saline were used as eluents. Fraction III and fraction IV $\left(\mathrm{K}_{\mathrm{av}}=0.68\right.$ and 0.95 , respectively) appeared to be rather heterogeneous accumulations of crude mucus-contaminating proteins with traces of carbohydrates.

In both lipomucoid and native gallbladder wall mucus, fraction II was the largest peak. Small quantities of high-molecular mass material, however, were eluted with the void volume. Lipomucoid proved to be highly enriched with lipids (see also 'Quantitative assays'). 
The lipids eluted with fraction II caused some light scattering. Defatted lipomucoid was eluted with peak I and II in a very similar way.

Human and pig gallbladder bile mucus showed similar elution profiles on Sephacryl S-300HR (fig. 3). The peak pattern differed significantly from that of fresh bile, in that fractions I and II were overlapped by a greatly increased fraction Ia which limited the separation efficiency. Pigment was associated with the material of fraction II.

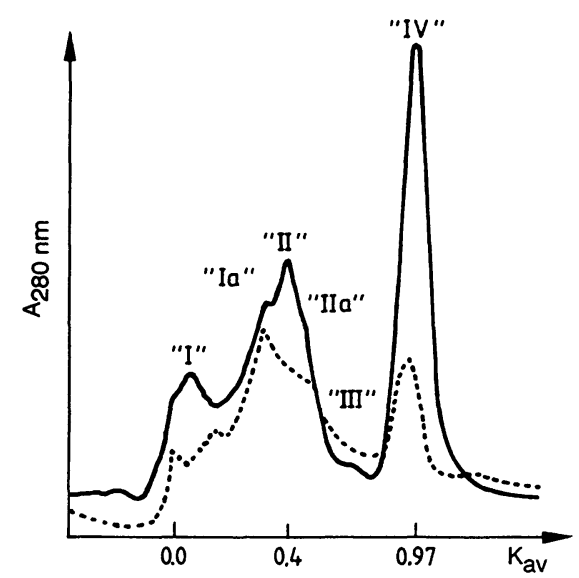

Fig. 3. Gel permeation elution profile.

Stationary phase: Sephacryl S-300 HR.

Eluent: $2 \mathrm{~mol} / \mathrm{l}$ urea, $10 \mathrm{~g} / 1 \mathrm{SB} 12$.

Sample:

— pig gallbladder bile mucus.

..... human gallbladder bile mucus.

Column: $35 \mathrm{~cm}$, ID $=1.0 \mathrm{~cm}$.

Flow rate: $3 \mathrm{ml} / \mathrm{h}$

"I"- "IV": areas of the fractions/peaks pointed out in fig. 2 .

Solubility, purification, and gelling properties

Although buffered physiological saline was free of detergents and the repeptized mucus did not form a clear solution, native mucus from bile (dissolved in water plus $0.4 \mathrm{~g} / 1 \mathrm{Na}$-azide) as well as crude total bile mucus did not flocculate during a $2-3$ month storage.

Crude total bile mucus/lipomucoid mixtures proved to be more stable than pure crude total bile mucus solutions. When crude total bile mucus dissolved in buffered physiological saline was additionally supplemented with urea $(2 \mathrm{~mol} / \mathrm{l})$ and SB12 $(10 \mathrm{~g} / \mathrm{l})$ and saturated with Na-tetraborate, the turbid solution became clear and opalescent after about $20 \mathrm{~h}$ at room temperature; this effect only occurred when lipomucoid was also present. When urea was removed by dialysis, fractions I and II flocculated in spite of the presence of SB12.
After ethanolic extraction of SB 12 and lyophilization, material of fractions I and II appeared as pure white spongy plugs, obviously free from pigment residues. It was now readily dissolvable in water, and the solution remained stable for about $40 \mathrm{~h}$. A smooth jelly was formed with $\sim 1 \mathrm{~g}$ of mucus per litre. Another fraction of pure white opalescent and pigment-free mucus material, obtained by precipitation from bile with acetic acid at $\mathrm{pH} 5.5$, repeatedly formed a clumped jelly at neutral $\mathrm{pH}$ in phosphate buffer. Its absorption at $\lambda=280 \mathrm{~nm}$ was extremely low. On gel permeation chromatography it was separated into an excluded and into a total volume fraction using buffered physiolgoical saline as an eluent. Methanolysis failed to access any carbohydrate portion. In contrast, acid precipitations, contaminated with some pigments, did not gelatinize but precipitated from the buffer. After extraction with $2 \mathrm{~mol} / 1$ urea/10 g/l SB12 (for removal of soluble mucoprotein), dialysis against distilled water, lyophilization, and ethanolic extraction (for removal of SB12), eluent insoluble mucus could be redissolved in water. It formed a thready and glassy gel which was irreversibly damaged by resolute shaking.

Determination of protein content, sugar and carbohydrate contents, and sialic acids contents

The protein contents of fractions I and II and of eluent insoluble mucus were comparable $(54.8,56.6$, and $59.4 \%$, respectively) and correlated well with the approximate $50 \%$ of protein found in pig gastric mucin (tab. 1). In contrast, defatted lipomucoid protein content was as low as expected in mucins $(\sim 30 \%$ of dry weight), while a portion of $70 \%$ remained unaccounted for (tab. 1).

The main neutral sugar measured in fraction I by capillary gas liquid chromatography was fucose, while galactose was predominant in fraction II, eluent insoluble mucus, and lipomucoid. Minor quantities of mannose were also measured in all mucus fractions. In fractions I and II the $\mathrm{N}$-acetyl glucosamine contents were three times higher than the $\mathrm{N}$-acetyl galactosamine contents; defatted lipomucoid and eluent insoluble mucus had only traces of hexosamines. The neutral carbohydrate : hexosamine ratio in fractions I and II was approximately $1: 1$ (in pig gastric mucin $=0.8: 1$ ). No uronic acids and no ribose could be detected, thus indicating the absence of glycosaminoglycans (keratan sulphate is not expected and was therefore disregarded) $)^{1}$ ) and of ribonucleic acids.

1) Although keratan sulphate, which is absent from uronic acid subunits, could be present in principle, it was not considered, since it has never been found in biliary mucus. 
Tab. 1. Chemical composition of porcine biliary mucus fractions $(\mathrm{mg} / \mathrm{kg})$

\begin{tabular}{|c|c|c|c|c|c|c|c|}
\hline \multirow{3}{*}{$\begin{array}{l}\text { Substance analysed/(method used) } \\
\text { Protein (20) } \\
\text { Carbohydrates (24) }\end{array}$} & Fraction I & \multicolumn{2}{|c|}{ Fraction II } & \multirow{2}{*}{$\begin{array}{l}\text { Defatted } \\
\text { lipomucoid }\end{array}$} & \multicolumn{2}{|c|}{$\begin{array}{l}\text { Eluent } \\
\text { insoluble } \\
\text { mucus }\end{array}$} & $\begin{array}{l}\text { Pig gastric } \\
\text { mucus (Sigma) }\end{array}$ \\
\hline & $548 \pm 69$ & $567 \pm$ & & & $594 \pm$ & & $475 \pm 2$ \\
\hline & 331 & 79 & & 47 & 60 & & 289 \\
\hline Neutral sugars & $163 \pm 6$ & $43 \pm$ & 2 & 47 & $60 \pm$ & 9 & $131 \pm 3$ \\
\hline Hexosamines & $167 \pm 13$ & $36 \pm$ & 3 & $\operatorname{tr}$ & $\operatorname{tr}$ & & $159 \pm 2$ \\
\hline Ratio neutral sugars/hexosamines & 10 & 12 & & - & - & & 8 \\
\hline \multicolumn{8}{|l|}{ Individual sugars } \\
\hline Fucose & $52 \pm 2$ & $9 \pm$ & 0 & 16 & $15 \pm$ & 2 & $35 \pm 1$ \\
\hline Mannose & $9 \pm 2$ & $8 \pm$ & 0 & 7 & $13 \pm$ & 2 & - \\
\hline Galactose & $7 \pm 4$ & $23 \pm$ & 3 & 24 & $23 \pm$ & 5 & $89 \pm 2$ \\
\hline Glucose & $9 \pm 1$ & - & & - & - & & $7 \pm 1$ \\
\hline $\mathrm{N}$-Acetyl galactosamine & $43 \pm 6$ & $8 \pm$ & 1 & $\operatorname{tr}$ & $\operatorname{tr}$ & & $71 \pm 2$ \\
\hline N-Acetyl glucosamine & $124 \pm 8$ & $31 \pm$ & 1 & $\operatorname{tr}$ & $\operatorname{tr}$ & & $88 \pm 4$ \\
\hline Sialic acids (23) & 32 & $15 \pm$ & 1 & ND & $12 \pm$ & 0 & $\mathrm{ND}^{-}$ \\
\hline Sialic acids (22) & $36 \pm 1$ & ND & & $13 \pm 2$ & $14 \pm$ & 1 & ND \\
\hline Lipids (19) & - & - & & - & $35 \pm$ & 2 & ND \\
\hline Total & 911 & 660 & & 309 & 701 & & 764 \\
\hline
\end{tabular}

Legend: Values represent means from repeated analyses $(\max n=9)$ except the values given for defatted lipomucoid carbohydrate measurement, which was a single experiment for lack of material; ND: not determined.

In our mucus preparations sialic acids could be oxidized under milder conditions than expected from experience, i.e. analysis of free sialic acids with the 'Acid Ninhydrin Test' (22) gave rise to higher values than those obtained with the 'Periodate-Resorcinol Test' (23) for total sialic acids. A modification of the latter (to measure covalently bound sialic acids under more rigorous oxidation conditions) destroyed $50 \%$ or more of neuraminic acids as calculated from the low recovery.

Besides carbohydrates and proteins, some fatty acids were found in eluent insoluble mucus ${ }^{2}$ ), which accounted for $\sim 3.5 \%$ of dry weight when calculated as phosphatidyl choline (tab. 1).

In summary: $91.1 \%$ of the chemical composition of fraction I, $66.0 \%$ of fraction II, $30.9 \%$ of defatted lipomucoid, and $70.1 \%$ of eluent insoluble mucus could be identified and measured. Cholesterol and phosphatidyl choline were the main lipids associated with mucus as revealed by thin layer chromatography. Minor quantities of lysophosphatidyl choline, phosphatidyl serine and phosphatidyl ethanolamine (or plasmalogens), and traces of esterified cholesterol were also present. The composition was similar to that of crude hen egg lecithin (Sigma), except that the phosphatidyl choline and cholesterol contents were even more dominant in mucus.

$\left.{ }^{2}\right)$ not ethanol washed

\section{Discussion}

While aiming at a mucolytic approach as the final of several steps, the first step should be the isolation and purification of mucoprotein complexes from bile mucus under mild conditions. Attempts to separate components of biliary mucus have already been made, starting from different assumptions and working under different conditions $(2-6,13,14)$. Of course, only those mucus portions have been taken in account that were easily soluble in physiological saline or in aqueous buffers, while poorly soluble components and dense gels have been separated by centrifugation and excluded from further investigations. According to the procedure of Pearson et al. (2) we first tried to remove the low molecular bile constituents by dialysis. This depended on whether we succeeded in separating the high viscous mucus gel from the pigments in the supernatant before dialysis, utilizing the effect of spontaneous stratification (native mucus from bile). Otherwise pigment-mucoprotein polymers were formed, which coagulated early during dialysis. By behaving in this way biliary mucus is likely to become an important constituent of pigment sludge, a bile sediment which was found to preceed pigment stone formation $(3,5)$.

As a rule we used the method of precipitation to make quite sure that we had obtained the total biliary mucoproteins, not being concerned with problems caused by large portions of pigment material, bile salts, and lipids. 
The further procedures with the four crude mucus preparations, i.e. gel permeation chromatography and several analytical tests, were performed under certain difficulties, due to the lability of mucus solubilizates in the analytical media. Mucus absorbance phenomena also played an important role.

Initial attempts to fractionate crude mucus using eluents described in the literature $(2,5)$ were unsatisfactory. Indeed we obtained comparable elution diagrams, but gross tailing and co-migration of the mucus components severely prevented good resolution and a neat separation. Moreover, the distinct affinity of samples for the stationary phase resulted in considerable losses of the product. Only when the experimental conditions were modified step by step, i.e. only when urea and SB12 were used as co-solvents and when the mucus samples were saturated with borate, did the separation became constant and the results were satisfactory.

The readiness with which mucoprotein forms stable solutions in the presence of both urea and SB12 is probably due to a reversible breaking up of intermolecular $\mathrm{H}$-bridges but also to an increased weakening of its hydrophobicity by polarization of hydrophobic domains. The added boric acid is able to link vicinal hydroxyl groups with cis-configuration, forming anions which, by themselves, may lower the hydrophobicity of the whole molecule. We should emphasize the finding that the stability of crude mucus solutions depended strongly on the portion of lipomucoid. It contains large amounts of the zwitterionic phosphatidyl choline, a substance with proved emulsifying properties. Referring to the effect of SB12, which is also a zwitterionic detergent, we speculate that SB12 functionally replaced the lecithin after most of the latter had been removed by acetone precipitation. Moreover, since SB12 is said to be a non-denaturing detergent (28) it is considered to be useful in restoring the nativity of the mucus fractions.

Since the main purpose of this investigation was a careful isolation, separation and purification of the mucoprotein complexes, while preserving them in the nearest native state as possible, further analyses (as described before) should be taken in the sense of group reactions, serving as a first gross characterization of single mucus fractions. We had to try numerous methods for analysis and quantitation until we found those which met the mucus requirements with respect to solvent $\mathrm{pH}$ and solubility enhancers. For instance, the commonly used periodate-Schiff-reagent (PAS) for glycoproteins (29) failed and we had to measure the carbohydrate content of mucus fractions by methanolysis and subsequent capillary gas liquid chromatographic monosaccharide quantitation. Methanolysis deacylates $\mathrm{N}$-acetyl hexosamines, thus making them indistinguishable from the unacetylated compounds, which could be present in the samples. But as almost all hexosamines incorporated in biological systems are acetylated we are justified in expressing the measured percentage of hexosamines as $\mathrm{N}$-acetyl hexosamines. Concerning protein quantitation, the well tried Coomassie method (30) proved to be badly reproducible; and only the Onishi modification (20) of the Lowry test (31), if handled with care (e. g. avoiding turbidity, precipitation, unstability of colour reaction) delivered reliable results. The elevated values for free sialic acids with the acid ninhydrin test (22), compared with the values for total sialic acids given by the periodate resorcinol test (23) may be explained by an interference of cysteine residues (22), which are surely present in the mucus.

The occurrence of mannose and glucose in the sugar moieties of fractions I, II, lipomucoid, and eluent insoluble mucus points to a close adherence of some serum proteins. A similar observation was made with commercial mucus preparations, e.g. with mucus from porcine stomach (Sigma) which was also found to contain glucose, in addition to the typical monosaccharide pattern of mucin glycoproteins (fucose, galactose, hexosamines).

Future work must be focused on the preparation of homogeneous mucin fractions as well as on the analysis of accompanying proteins. We have to investigate possible interactions of mucin macromolecules with bile ingredients, especially with those involved in the formation of gallstones. Smith \& LaMont (5), for instance, demonstrated in vitro that biliary mucus and bilirubin formed a complex. Such a complex is expected to impede chemolitholysis.

It remains to state that the fundamentals of a mucolytic approach to local chemolitholysis can only be worked out experimentally. An adequate mucus model is considered essential, and the present work shows that such a model is available. 


\section{References}

1. Nakamura, N. (1967) Mucopolysaccharides and their significance in gallstone formation. Tohoku J. Exp. Med. 93, $235-247$.

2. Pearson, J. P., Kaura, R., Taylor, W. \& Allen, A. (1982) The composition and polymeric structure of mucus glycoprotein from human gallbladder bile. Biochim. Biophys. Acta $706,221-228$.

3. Smith, B. F. \& LaMont, J. T. (1985) Identification of gallbladder mucin-bilirubin complex in human cholesterol gallstone matrix. Clin. Chim. Invest. 76, 439-445.

4. LaMont, J. T., Ventola, A. S., Trotman, B. W. \& Soloway, R. D. (1983) Mucin glycoprotein content of human pigment gallstones. Hepatology 3, 377-382.

5. Smith, B. F. \& LaMont, A. S. (1983) Bovine gallbladder mucin binds bilirubin in vitro. Gastroenterology 85, $707-$ 712.

6. Smith, B. F., Peetermans, J. A., Tanaka, T., LaMont, J. T. (1989) Subunit interactions and physical properties of bovine gallbladder mucin. Gastroenterology 97, 179-187.

7. LaMont, J. T. \& Turner, B. (1981) Role of gallbladder mucus in formation of gallstone: Organ culture studies. NIH International Workshop of Pigment Gallstone Disease, Univ. of Pennsylvania, May 13-15, 1981.

8. LaMorte, W. W., Brotschi, F. A., Scott, Th. F. \& Williams jr., L. F. (1985) Pigment gallstone formation in the cholesterol-fed guinea pig. Hepatology 5, 21-27.

9. LaMorte, W. W., LaMont, J. T., Hale, W., Booker, M. L., Scott, T. E. \& Turner, B. (1986) Gallbladder prostaglandins and lysophospholipids as mediators of mucin secretion during cholelithiasis. J. Amer. Physiol. 251 G701-G710.

10. Bernhoft, R. A., Pellegrini, C. A., Broderick, W. C. \& Way, I. W. (1983) Pigment sludge and stone formation in the acutely ligated dog gallbladder. Gastroenterology 85 , $1166-1171$

11. Lee, S. P. (1981) Hypersecretion of mucus glycoprotein by the gallbladder epithelium in experimental cholelithiasis. J. Pathol. 134, 199-207.

12. Good, L. I., Trotman, B. W. \& Soloway, R. D. (1978) Protein content of pigment gallstones. Role in pathogenesis. Gastroenterology 74, 1125.

13. Lee, S. P., Lim, T. H. \& Scott, A. J. (1979) Carbohydrate moieties of glycoproteins in human hepatic and gallbladder bile, gallbladder mucosa and gallstones. Clin. Sci. 56, 533538.

14. Maki, T., Matsushiro, T., Suzuki, N. \& Nakamura, N. (1971) Role of sulfated glycoprotein in gallstone formation. Surg. Gynaecol. Obstet. 132, 846-854.

15. Callaghan, R. M. (1989) Characterization of human tracheobronchial mucin glycoproteins. Methods Enzymol. 179, $3-17$.

16. Kleimann, H. K., Mc Garvey, M. L., Hassell, J. R., Star, V. L., Cannon, B. F., Laurie. G. W. \& Martin, J. L. (1986) Basement membrane complexes with biological activity. Biochemistry 25, 312-318.
17. Smith, B. F. (1987) Human gallbladder mucin binds biliary lipids and promotes cholesterol crystal nucleation in model bile. J. Lip. Res. 28, 1088-1098.

18. Hansson, G. C., Sheehan, J. K. \& Carlstedt, I. (1988) Only trace amounts of fatty acids are found in pure mucus glycoproteins. Arch. Biochem. Biophys. 266, 197-200.

19. Sabinski, F., Barckhaus, R. H., Fromme, H. G. \& Spener, F. (1982) Dynamics of galactolipids and plastids in nonphotosynthetic cells of Glycine Max suspension cultures. Plant. Physiol. 70, 610-615.

20. Onishi, S. T. \& Barr, J. K. (1978) A simplified method of quantitating protein using the biuret and phenol reagent. Anal. Biochem. 86, 193-200.

21. de Jong, J. G. N., Wevers, R. A., Laarakkers, C. \& Porthuis, B. J. H. M. (1989) Dimethylene blue-based spectrophotometry of glycosaminoglycans in untreated urine. A rapid screening procedure for mucopolysaccharidosis. Clin. Chem. 35, 1472-1477.

22. Yao, K. \& Ubuka, T. (1987) Determination of sialic acids by acetic ninhydrin reaction. Acta Med. Okayama 41, $237-$ 241.

23. Jourdian, G. W., Dean, L. \& Roseman, S. (1971) The sialic acids. XI A periodate resorcinol method for the quantitative estimation of free sialic acids and their glycosides. J. Biol. Chem. 246, 430-435.

24. Sabinski, F. \& Wosiewitz, U. (1984) The sugar spectrum of human cholesterol stones, mixed and pigment gallstones: combined quantitative analysis of neutral sugars, $\mathrm{N}$-acetyl hexosamines, hexuronic acids, and $\mathrm{N}$-acetyl neuraminic acids by capillary gas-liquid chromatography. J. Clin. Chem. Clin. Biochem. 22, 453-459.

25. Mantle, M. \& Allen, A. (1981) Isolation and characterization of native glycoprotein from pig small-intestinal mucus. Biochem. J. 195, 267-275.

26. Starkey, B. J., Snary, D. \& Allen, A. (1974) Characterization of gastric mucoproteins isolated by equilibrium density-gradient centrifugation in caesium chloride. Biochem. J. $141,633-639$.

27. Smith, B. F. (1987) Dissolution of cholesterol gallstones in vitro. Gastroenterology 93, 98-105.

28. Gonenne, A. \& Ernst, R. (1978) Solubilization of membrane proteins by sulfobetains, novel zwitterionic surfactants. Anal. Biochem. 87, 28-38.

29. Mantle, M. \& Allen, A. (1987) A colorimetric assay for glycoproteins based on the periodic acid/Schiff stain. Biochem. Soc. Transact. 6, 607-609.

30. Bradford, M. M. (1976) A rapid and sensitive method for the quantitation of microgram quantities of protein utilizing the principle of protein-dye binding. Anal. Biochem. $72,248-254$.

31. Lowry, O. H., Rosebrough, N. J., Farr, A. L. \& Randall, R. J. (1951) Protein measurement with the Folin phenol reagent. Biol. Chem. 193, 265-275.

\section{Dr. F. Sabinski}

Institut f. Med. Physik u. Biophysik der Westfälischen Wilhelms-Universität

Hüfferstraße 68

W-4400 Münster

Bundesrepublik Deutschland 
